\title{
Electronic structure and layer-resolved transmission of bilayer graphene nanoribbon in the presence of vertical fields
}

\author{
Habib Rostami ${ }^{1}$ and Reza Asgari ${ }^{1, *}$ \\ ${ }^{1}$ School of Physics, Institute for Research in Fundamental Sciences (IPM), Tehran 19395-5531, Iran
}

(Dated: June 7, 2021)

\begin{abstract}
Electronic properties of bilayer graphene are distinct from both the conventional two dimensional electron gas and monolayer graphene due to its particular chiral properties and excitation charge carrier dispersions. We study the effect of strain on the electronic structure, the edge states and charge transport of bilayer graphene nanoribbon at zero temperature. We demonstrate a valley polarized quantum Hall effect in biased bilayer graphene when the system is subjected to a perpendicular magnetic field. In this system a topological phase transition from a quantum valley Hall to a valley polarized quantum Hall phase can occur by tuning the interplanar strain. Furthermore, we study the layer-resolved transport properties by calculating the layer polarized quantity by using the recursive Green's function technique and show that the resulting layer polarized value confirms the obtained phases. These predictions can be verified by experiments and our results demonstrate the possibility for exploiting strained bilayer graphene in the presence of external fields for electronics and valleytronics devices.
\end{abstract}

PACS numbers: 73.22.Pr, 62.20.-x, 72.10.Di, 71.70.Di

\section{INTRODUCTION}

Crystalline bilayer graphene (BLG) $)^{1-3}$ has recently attracted a great deal of attention because of its unique electronic properties. ${ }^{4}$ It consists of two single-layer graphene (SLG) sheets in which the second carbon layer is rotated by $60^{\circ}$ with respect to the first one, separated by a small distance and can be produced by mechanical exfoliation of thin graphite or by thermal decomposition of silicon carbide. ${ }^{5}$ The low-energy quasiparticles in BLG behave as massive chiral fermions and are responsible for a plethora of interesting physics including broken-symmetry states at very weak magnetic fields when BLG is suspended to reduce disorder ${ }^{6}$ and anomalous exciton condensation in the quantum Hall regime. ${ }^{7}$ Although the intrinsic BLG is a zero-gap semi-metal, it becomes a tunable band gap semiconductor ${ }^{8,9}$ when a gate voltage is applied. Much of this attraction is due to its structure having two Dirac points in the Brillouin zone (BZ), known as $\boldsymbol{K}$ and $\boldsymbol{K}^{\prime}$.

Many interesting properties emerge in strained BLG systems in analogue of monolayer graphene sheet. ${ }^{10-16}$ It has been shown that homogeneous strain profoundly changes the topology of band structure and the Lifshitz transition takes place in strained BLG upon splitting the parabolic bands at intermediate energies into several Dirac cones and consequently this affects the electron Landau level spectra and the quantum Hall effect. ${ }^{17}$ Moreover, in uniaxially strained BLG, each $\boldsymbol{K}$-point splits into two pockets. ${ }^{18}$ The size of the energy gap can be controlled by adjusting the strength and direction of different homogeneous strains in BLG. ${ }^{19-21}$ The effect of interplanar strain on the electronic structure of the bilayer graphene in the presence of an electric field has been studied by Nanda and Satpathy ${ }^{22}$ within $a b$ inito calculations. They found that while strain alone does not produce a gap, an electric field does so in the
Bernel structure but not in the hexagonal structure. Recently, a theoretical study in bilayer graphene nanoribbon (BLGNR) shows that a mechanical deformation can induce effective fields which modifies the dynamical behavior of electrons. ${ }^{23}$ Interestingly, the creation of pseudomagnetic field in a Moirè pattern of a twisted BLG has also been reported by Yan and et al. ${ }^{24}$ and recently analyzed in STS measurement. ${ }^{25}$ A large body of the studies is based on homogeneously strained BLG even though inhomogeneous strain needs specific care.

One of the interesting phenomena that has been observed in bilayer is the anomalous quantum Hall effect. The quantum Hall effect can be understood by solving the quantum-mechanical problem for the bulk of the system in the presence of a magnetic field. There is an alternative approach to the quantum Hall effect that is actually based on the analysis of the edge states of electrons in a magnetic field. ${ }^{26}$ These edge states are chiral since only one direction of propagation is allowed. If thus all bulk states are localized there is still a current being carried by electrons on the boundary with a contribution to conductance. BLG is an interesting system to study since its high magnetic field Landau level consists of equidistant groups of fourfold degenerate state at finite energy and supports eight zero-energy states and can be broken by perpendicular electric field. ${ }^{7,27}$ This helps to control the edge state structure using a combination of the electric field and the magnetic field too.

In the BLG, there are four carbon atoms per unit cell and it turns out that there is also (at least for the case that energies are below the interlayer bonding energy $\left.\gamma_{1}\right)$ a spin $1 / 2$ pseudospin degree of freedom can be interpreted as labeling a layer rather than a sublattice. Since the sublattice pseudospin is equivalent to a layer, an electric field perpendicular to layers couples to the pseudospin degree of freedom much stronger than a practical magnetic field coupled to the real spin. This 
surprising feature of the electronic properties of BLG offers a possible potential for digital electronics based on graphene flakes. ${ }^{28-30}$ Moreover, in the BLG system there is a length scale corresponding to the interlayer hopping that indicates the traveling length of electrons between the interlayer hopping and it is about $l_{\perp} \approx 11 a_{0}$ with $a_{0}=0.142 \mathrm{~nm}$. Therefore, a layer-flip relaxation time $\tau_{L f}$ should be defined in analogue with a spin-flip relaxation time in non-collinear spin dependence transport. Typical length of bilayer device is much longer than the $l_{\perp}$, in order that two layers are strongly coupled. ${ }^{31}$

The general features of the electronic structure, basically the structure of the Landau level and edge states in the quantum Hall regime, have been numerically stud$\operatorname{ied}^{32}$ for the unbiased and unstrained BLGNR. In the present work we study the effect of strain on the electronic structure, particulary the edge states and charge transport, of the BLGNR system at zero temperature. In the system, similar to monolayer graphene, a pseudomagnetic field can be created. We first propose a lattice model Hamiltonian to explore BLG under the combined effect of deformations, real magnetic fields and gate voltages. The lattice model Hamiltonian, which we propose, provides a quite good description of the edge states since it incorporates the correct expressions of the hopping integrals when the system is deformed and we show that pseudo Landau levels in the bulk are no longer dispersive. We study comprehensively the edge states inside the gap (created by vertical bias) in the presence of a real and pseudomagnetic fields and explore different phases in BLGNR for the different values of the interlayer spacing. We obtain a valley polarized quantum Hall effect in biased bilayer graphene when the system is subjected to a perpendicular real magnetic field for certain values of the interlayer hopping integral. We show that, in stained and biased BLGNR exposed by a magnetic field, a topological phase transition from a quantum valley Hall to a valley polarized quantum Hall can occur by tuning the interlayer spacing (interplanar strain) between the two layers. In different phases, the number of conducting channel is calculated by recursive Green's function approach together with the Landauer formalism and the conductance feature supports the existence of those phases. Moreover, we investigate the effect of the interlayer spacing on the layer-resolved transport in BLGNR by using an analog between layer and spin-resolved transport physics. ${ }^{33}$

The paper is organized as follows. In Sec. II we introduce the formalism that will be used in calculating the electronic structure, two terminal conductance and the layer polarization quantity from the recursive Green's function approach. In Sec. III we present our analytic and numeric results for the dispersion relation in both strained and unstrained biased bilayer graphene sheets in the presence of the magnetic field. Section IV contains a brief summary of our main results.

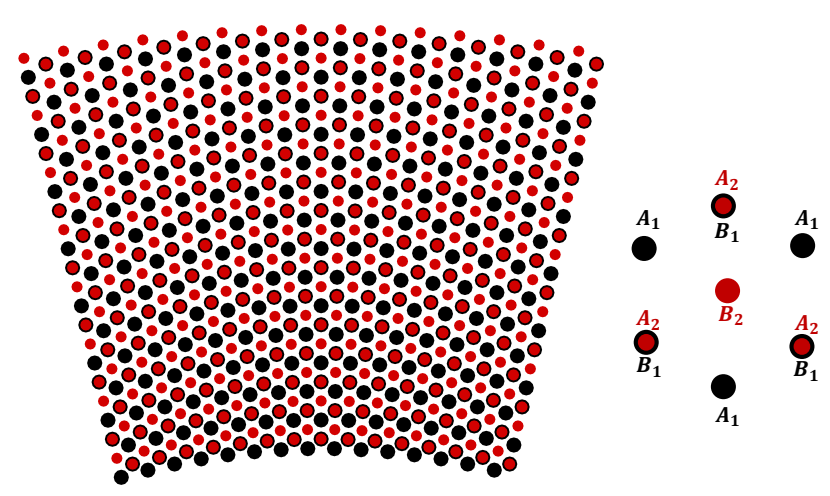

FIG. 1. (Color online) Sketch of an arc-shaped bending bilayer graphene lattice and sublattice labels in a Bernalstacking and the top view of the positions of carbon atoms.

\section{THEORY AND MODEL}

Bilayer graphene is a crystal system consisting of two single layers of graphene sheet offset from each other in the $x y$ plane. The top $A$ sublattice is directly above the bottom $B$ sublattice and it is between these pairs of atoms that the interlayer dimer bonds are formed, whereas there are no essential hopping processes between a counterpart on the other layer. We consider an arcshaped bending BLGNR with zigzag edges to explore the electronic structures in the presence of perpendicular fields. We assume that the $s p^{2}$-hybridized electrons of carbon atoms in each sheet are inert and only take into account the $2 p_{z}$ electrons which form the $\pi$ bands. A top view of the system is displayed in Fig. 1 and hopping parameters are defined as $t_{A_{1}, B_{1}}=t_{A_{2}, B_{2}}=\gamma_{0}$, $t_{B_{1}, A_{2}}=\gamma_{1}, t_{A_{1}, B_{2}}=\gamma_{3}$ and $t_{A_{1}, A_{2}}=\gamma_{4}$.

Most of the interesting properties of the Bernelstacking BLGNR can be understood in the framework of a tight-binding model Hamiltonian. ${ }^{7}$ In deformed graphene system, the ideal atomic configurations change and all bonds of each atom with its neighbors are no longer equivalent and therefore the hopping parameters are different throughout the whole sample. We modify the tight-binding model for a deformed BLGNR by taking into account atomistic inhomogeneities, and we find the following effective Hamiltonian

$$
\begin{aligned}
H & =\sum_{i, l}\left\{\epsilon_{i}{ }^{i} a_{i}^{l^{\dagger}} a_{i}^{l}+\epsilon_{i}{ }^{b} b_{i}^{l^{\dagger}} b_{i}^{l}\right\} \\
& -\sum_{<i j>, l}\left\{\gamma_{0}^{i j} e^{\phi_{i j}} a_{i}^{l^{\dagger}} b_{j}^{l}+H . c .\right\} \\
& -\sum_{i}\left\{\gamma_{1}^{i j} a_{i}^{2^{\dagger}} b_{i}^{1}+H . c .\right\} \\
& -\sum_{<i j>}\left\{\gamma_{3}^{i j} e^{\phi_{i j}} a_{i}^{1 \dagger} b_{j}^{2}+H . c .\right\}
\end{aligned}
$$


where we define ${ }^{34,35,37}$

$$
\begin{aligned}
& \gamma_{0}^{i j}=\gamma_{0} e^{-\beta_{0}\left(\frac{d}{a_{0}}-1\right)}, \quad \gamma_{1}^{i j}=\gamma_{1} e^{-\beta_{1}\left(\frac{d}{c_{0}}-1\right)} \\
& \gamma_{3}^{i j}=X\left[\gamma_{0}\left(\frac{d_{\|}}{d}\right)^{2} e^{-\beta_{0}\left(\frac{d}{c_{0}}-1\right)}+\gamma_{1}\left(\frac{d_{\perp}}{d}\right)^{2} e^{-\beta_{1}\left(\frac{d}{\bar{c}_{0}}-1\right)}\right]
\end{aligned}
$$

with $\gamma_{0}=-3.16 \mathrm{eV}, \gamma_{1}=0.39 \mathrm{eV}, a_{0}=0.142 \mathrm{~nm}$, $c_{0}=0.335 \mathrm{~nm}, \tilde{c}_{0}=\sqrt{a_{0}^{2}+c_{0}^{2}}=0.364 \mathrm{~nm}, \beta_{0}=2.9$, $\beta_{1}=c_{0} \beta_{0} / a_{0}=6.8$ and $d=\left|\mathbf{r}_{i}-\mathbf{r}_{j}\right|$ which has inplane $\left(d_{\|}\right)$and vertical $\left(d_{\perp}\right)$ components. $\gamma_{3}^{i j}$ in Eq. (2) results from the Slater-Koster table ${ }^{36}$ with an empirical parameter. Setting $X=1.76$, it generates a reasonable hopping energy for an ideal BLG with $\gamma_{3}=0.315 \mathrm{eV}$. In addition, $\epsilon_{i}^{a(b)}$ is the on site potential at point $A(B)$. In a clean ribbon, we have $\epsilon_{i}=(-1)^{l} u / 2$ where $u$ stands for induced potential due to external perpendicular electric field which breaks layer degeneracy and $l=1,2$ stands to the layer number. $\phi_{i j}=\frac{e}{\hbar} \int_{i}^{j} \vec{A} \cdot \overrightarrow{d r}$ is the Peierls phase factor to carry out the orbital effect of the perpendicular real magnetic field. Another hopping energy between the nearest-neighboring layers, $\gamma_{4}=0.04 \mathrm{eV}$, is very small compare to $\gamma_{0}$ and can be ignored.

It would be worthwhile mentioning that the exponential correction of the hopping integrals $\left(\beta_{0}\right.$ term) is consistent with experimental results ${ }^{38}$ of SLG in which $\partial \gamma_{0} / \partial a_{0}=-6.4 \mathrm{eV}^{-1}$ and it provides quite good descriptions of the system with respect to those results obtained within Harrison's approach ${ }^{39}$ in which $\gamma_{0(1)}^{i j}=$ $\gamma_{0(1)}\left[1-\beta_{0(1)} \boldsymbol{d}_{0} \cdot\left(\boldsymbol{d}-\boldsymbol{d}_{0}\right) / d_{0}\right]$ where $\beta_{0(1)} \approx 2$ and $\boldsymbol{d}_{0}$ is the distance between two atoms in unstrained graphene. We will discuss the discrepancy of these approaches in the next Section and show that pseudo Landau levels in the bulk are no longer dispersive by using the exponential correction of the hopping integrals.

In the absence of strain and external magnetic field the $\boldsymbol{k}$-space Hamiltonian in four dimensional sublattice space of $\left(A_{1}, B_{2}, A_{2}, B_{1}\right)$ can be written as follows

$$
\mathcal{H}=\left(\begin{array}{cc}
\mathcal{H}_{1} & \mathcal{V} \\
\mathcal{V}^{\dagger} & \mathcal{H}_{2}
\end{array}\right)
$$

where the interlayer and intralayer contributions are $\mathcal{H}_{i=1,2}=U_{i} \sigma_{0}+\hbar v_{0}\left(\sigma_{x} q_{x}+\sigma_{y} q_{y}\right)$ and $2 \mathcal{V}=\gamma_{1} \sigma_{-}+$ $v_{3}\left(q_{x}+i q_{y}\right) \sigma_{+}$, respectively. Here $\sigma_{ \pm}=\sigma_{x} i \pm \sigma_{y}$ with Pauli matrix $\sigma_{i}$. This Hamiltonian includes a noncollinear physics for the layer degree of freedom which it can be assumed as an pseudospin in BLG. ${ }^{28} \mathrm{~A}$ general Hamiltonian for the deformed BLG Hamiltonian in the $\boldsymbol{k}$-space is quite cumbersome ${ }^{23}$ however, in the lowenergy limit, it can be simplified for some special cases. For instance, when the deformation is just in-plane where two layers are deformed in a same way, the two-band Hamiltonian can be written as

$$
\begin{aligned}
H_{k} & =-\frac{v_{0}^{2}}{\gamma_{1}}\left(\begin{array}{cc}
0 & (\mathbf{p}-e \mathbf{A})^{\dagger^{2}} \\
(\mathbf{p}-e \mathbf{A})^{2} & 0
\end{array}\right) \\
& +\left(\begin{array}{cc}
\frac{u}{2} & v_{3}\left(\mathbf{p}-e \eta_{3} \mathbf{A}\right) \\
v_{3}\left(\mathbf{p}-e \eta_{3} \mathbf{A}\right)^{\dagger} & -\frac{u}{2} .
\end{array}\right)
\end{aligned}
$$

Here $v_{0}=3 \gamma_{0} a_{0} / 2 \hbar, \quad v_{3}=3 \gamma_{3} a_{0} / 2 \hbar, \quad \eta_{3}=$ $2\left(a_{0} / \tilde{c}_{0}\right)^{2} \beta_{3} / \beta_{0}, \quad \beta_{0}=-\partial \log \gamma_{0} / \partial \log a_{0}$ and $\beta_{3}=$ $-\partial \log \gamma_{3} / \partial \log \tilde{c}_{0}$. The fictitious gauge filed, therefore, is defined as $\mathbf{A}=\frac{\phi_{0}}{a_{0}} \frac{\beta_{0}}{2 \pi}\left(\varepsilon_{x x}-\varepsilon_{y y},-2 \varepsilon_{x y}\right)$. Notice that $\eta_{3} \approx 2$ based on our model. In this paper, however, we would like to consider more general cases and thus we use Eq. (1) in the real space Hamiltonian and find its associated Hamiltonian in the $\boldsymbol{k}$-space. In ribbon geometry, in other words, it is easy to find the energy dispersion relation with the periodic boundary condition along the ribbon in the $x$-direction. To do so, we assume an infinite stack of principal layers with the nearest-neighbor interactions. A principle layer is defined as the smallest group of neighboring atoms planes in such a way that only nearest-neighbor interactions exist between principle layers. Thus, we can transform the original system into a linear chain of the principal layers. ${ }^{43}$ Owing to the translational invariant along $x$, the momentum in the $x$-direction is a good quantum number. To study the band structure properties provided by our tight-binding model, we find its $\boldsymbol{k}$-space forms as $\sum_{\boldsymbol{k}} \psi_{\boldsymbol{k}}^{\dagger} H_{k} \psi_{\boldsymbol{k}}$. After performing the Fourier transformation along the $x$-direction, the Hamiltonian in $\boldsymbol{k}$-space can be written as

$$
H_{k}=H_{00}+H_{01} e^{-i k_{x} a}+H_{01}^{\dagger} e^{i k_{x} a}
$$

in which $a=\sqrt{3} a_{0}$. Moreover, $H_{00}$ and $H_{01}$ describe coupling within the principal layer (intra layer) and the adjacent principal layers (inter layer), respectively based on the tight-binding model given by Eq. (1). This Hamiltonian can be simply diagonalized and thus the energy dispersion of a ribbon in the presence of a real and pseudomagnetic fields can be obtained. Furthermore, corresponding wave function for a given energy and wave vector can be used in order to calculate the siteresolved local density of states (LDOS) for the ribbon as $\rho\left(y, E_{n k}\right)=\sum_{m k^{\prime}}\left|\psi_{m k^{\prime}}(y)\right|^{2} \delta\left(E_{n k}-E_{m k^{\prime}}\right)$ where $n, m$ are band indexes.

In general, strain can induce a scalar potential as a diagonal term ${ }^{23}$ and it originates from a redistribution of electron density under the deformation. Since the scalar potential suppresses drastically due to the effect of electron screening ${ }^{40}$ and describes only the electron profile on the BLG, we thus ignore the scalar potential in our calculations. We assume an arc-shape deformation in order to produce fictitious gauge field in which the atomic displacement profile is $\left(u_{x}, u_{y}\right)=\left(x y / R,-x^{2} / 2 R\right)$ in both layers. This deformation leads to a constant pseudomagnetic field with opposite sign in two valleys as $\left|B^{p s}\right|=\phi_{0} \beta_{0} / 2 \pi a_{0} R$ (by neglecting $\gamma_{3}$ ) with corresponding magnetic length $l_{B}^{p s}=\sqrt{2 a_{0} R / \beta_{0}}$. Here, $R$ is the bending radius of the deformation applied to the flakes.

Importantly, the fictitious gauge field appearing in the arc-shaped in BLGNR differs from that created in the arc-shaped bending SLG nanoribbon due to the interlayer hopping contributions. The concept of the pseudomagnetic field is still well-defined in BLGNR as well since the interlayer contribution of the fictitious gauge 
field is weaker than its counterpart for the intralayer hopping. Moreover, the variations of $\gamma_{1}$ is controllable by a perpendicular force which changes the interlayer spacing without generating any pseudomagnetic field. In the vertical direction, the mechanical strength generates from a weak $\sigma$-bond between $p_{z}$ orbitals of carbon atoms, we can thus change the interlayer spacing in a wide range without breaking the symmetries of the system. We reveal the effect of the interlayer hopping values by looking at the physics of the edge states in the system.

To calculate conductance we use the non-equilibrium Green's function method in which retarded Green's function is defined as $G=\left(E-H-\Sigma+i 0^{+}\right)^{-1}$ by employing the recursive Green's function method. ${ }^{41}$ In noninteracting Hamiltonian the self-energy $\left(\Sigma=\Sigma_{L}+\Sigma_{R}\right)$ only originates from the connection of the system to the leads and it can be calculated by a method that has been developed and implemented for BLGNR. ${ }^{42,43}$ Transmission function, $T$ is obtained from Green's function and line width function $\Gamma_{L, R}$ too. ${ }^{43}$ The Landauer formula then gives us the zero temperature conductance, $2 e^{2} T / h$. In order to study the layer-resolved transport of BLGNR, we have designed an electronic system in which leads are modelled as two decoupled monolayer graphene. In this case $\Gamma_{L, R}$ are block diagonal matrixes in layer-space and therefore layer-resolved transmission components at zero temperature can be read as follows

$$
\begin{aligned}
& T^{\sigma \sigma^{\prime}}=\operatorname{Tr}\left[\Gamma_{L}^{\sigma \sigma} G^{\sigma \sigma^{\prime}} \Gamma_{R}^{\sigma^{\prime} \sigma^{\prime}} G^{\dagger \sigma^{\prime} \sigma}\right] \\
& \Gamma_{L, R}=-2 \Im m\left[\Sigma_{L, R}\right]
\end{aligned}
$$

where $\sigma, \sigma^{\prime}$ correspond to the layer degree of freedom and being 1 or 2 . In Landaure formalism, zero-temperature conductance in a two terminal setup is given by

$$
G=\frac{2 e^{2}}{h}\left(T_{L c}+T_{L f}\right)
$$

where layer-conserve and layer-flip transmissions are defined as $T_{L c}=T^{11}+T^{22}$ and $T_{L f}=T^{12}+T^{21}$, respectively. These definitions are given in a similar way that the spin-resolved components have been specified. ${ }^{33}$ We thus define the layer polarization, in analog with non-collinear spin-dependent system, as $P=$ $\left(T_{L c}-T_{L f}\right) /\left(T_{L c}+T_{L f}\right)$ which can be valued between 1 and -1 corresponding to a completely layer-conserve and layer-flip transport cases, respectively. In order to count the number of transport channels, we assume the same structure of the leads and scattering region in order that unexpected scattering can be eliminated in the interface of the leads and scattering region.

\section{NUMERICAL RESULTS AND DISCUSSIONS}

In this section, we present our main calculations for the electronic properties of BLGNR by evaluating Eqs. (1),
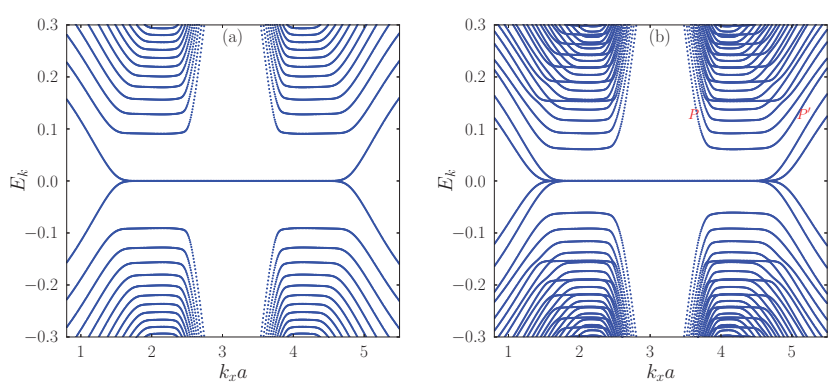

FIG. 2. (Color online) Pseudo Landau level in a SLG nanoribbon (a) and BLGNR (b) as a function of $k_{x} a$ for the ribbon width $W=300.5 a_{0}$ and the bending radius $R=601 a_{0}$. The PLLs are no longer dispersive since the exponential hopping correction used in our model results in flat PLLs. The zigzag boundary condition do not mix the Dirac points leading to a complete distinction of the edge modes. Two edge states on two edges of the arc-shaped graphene are no longer the same ( labelled by $p$ and $p^{\prime}$ in (b)) thus quasiparticles in one edge move faster than one along another edge.
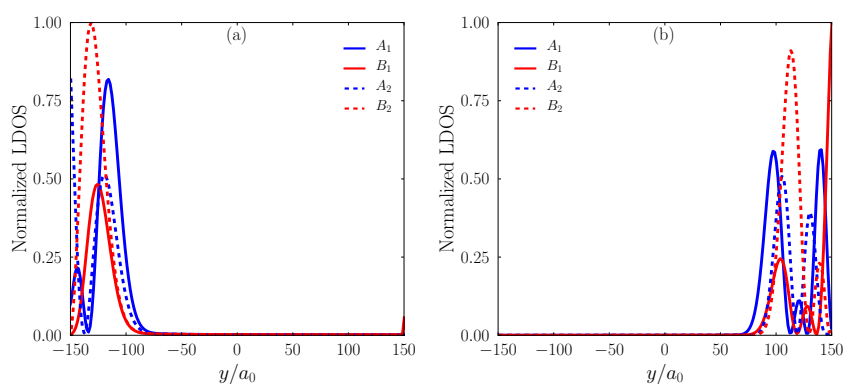

FIG. 3. (Color online) Site-resolved normalized local density of states (LDOS) as a function of distance for PLLs for P (a) and $P^{\prime}$ (b) edge state indicated in Fig. 2 b for $E=0.13 t_{0}$, $k_{P}=3.7$ and $k_{p^{\prime}}=5.1$. Numerical results show that the faster moving particle on edge is localized on the compressed edge and the slower one is located on the stretched edge.

(5) and (6). The general features of the electronic structure, basically the structure of the Landau level and edge states in the quantum Hall regime, have been numerically studied previously ${ }^{32}$ for the unbiased and unstrained BLGNR. We first modify the lattice Hamiltonian by using correct expressions of the hopping integrals for strained graphene. Second, we present our comprehensive numerical results of electronic structure by exploring the edge states and the layer-resolved transport properties of biased BLGNR in two different interesting regimes an ideal and a deformed bilayer graphene.

To investigate the generation of pseudomagnetic field in the arc-shaped bending SLG and BLG nanoribbon systems we first calculate the electronic spectrum shown in Fig. 2 in which the flat pseudo Landau levels (PLLs) can be clearly obtained. It should be noticed that PLLs in the bulk are not dispersive and our numerical results 


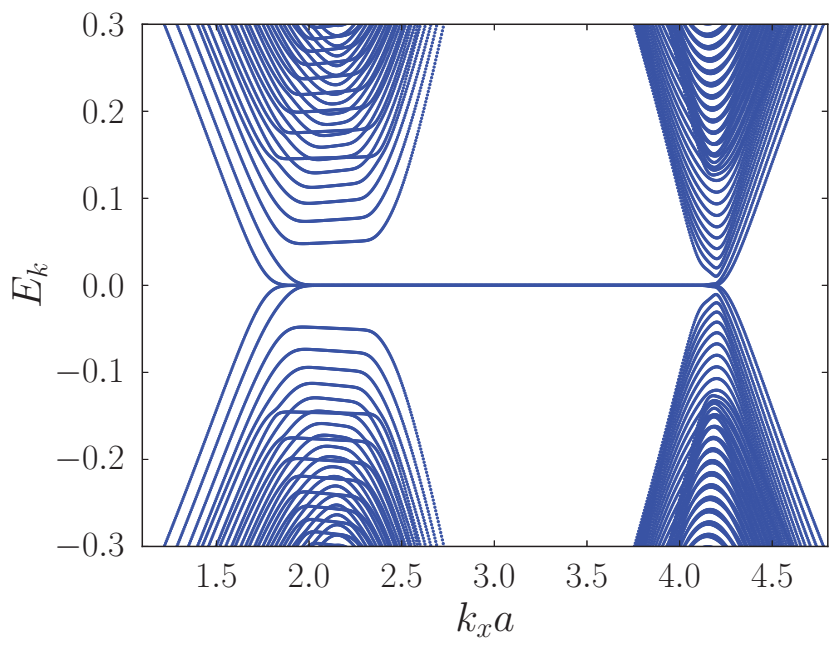

FIG. 4. (Color online) Energy dispersion in an arc-shaped bending BLGNR in a real magnetic field as a function of $k_{x} a$ for $W=300.5 a_{0}$, the bending radius $R=1803 a_{0}$ and the pseudomagnetic length $l_{B}=40.5 a_{0}$. Clear quantum Hall plateaus are observed mimicking the conventional quantum Hall effect in BLG. The combination of the pseudomagnetic field due to deforming and a real magnetic field leads to the effective total fields acting on electrons from the valleys $\boldsymbol{K}$ and $\boldsymbol{K}^{\prime}$ being different which results in a valley polarization.

differ slightly from dispersive PLLs results reported in Refs. [44,45]. Dispersive Landau levels are just expected in the system when it is subjected by an electric field which generates a drift velocity of charge carriers through the edge of the system. ${ }^{46}$ The reason for the discrepancy mainly arises from the different form of the hopping corrections. Based on our numerical calculation, the Harrison's method results in dispersive PLLs ${ }^{44,45}$ however the exponential hopping correction that we have implemented in our model results in flat PLLs. The spectrum in both SLG and BLG nanorribbon systems is not equidistance. The zigzag boundary condition does not mix the Dirac points leading to a complete distinction of the edge modes. Furthermore, the zero Landau level with zero-energy is chiral and topologically protected. Meanwhile, two edge states on the two edges of the arc-shaped graphene are no longer the same (labeled by $p$ and $p^{\prime}$ in Fig. $2 b)$ so that quasiparticles in one edge move faster than one along other edge. This difference arises from compressing and stretching strain in the bottom and top edges respectively and as a result the velocity of quasiparticle in the compressed edge is larger than the one in the stretched edge. ${ }^{16}$ In order to clarify this analysis, we thus show in Fig. 3 the site-resolved local density of state of those edge states and our numerical results confirm that the faster moving particle on edges is localized on the compressed edge and the slower one is located on the stretched edge.

Next, we examine the interplay between real and pseu-
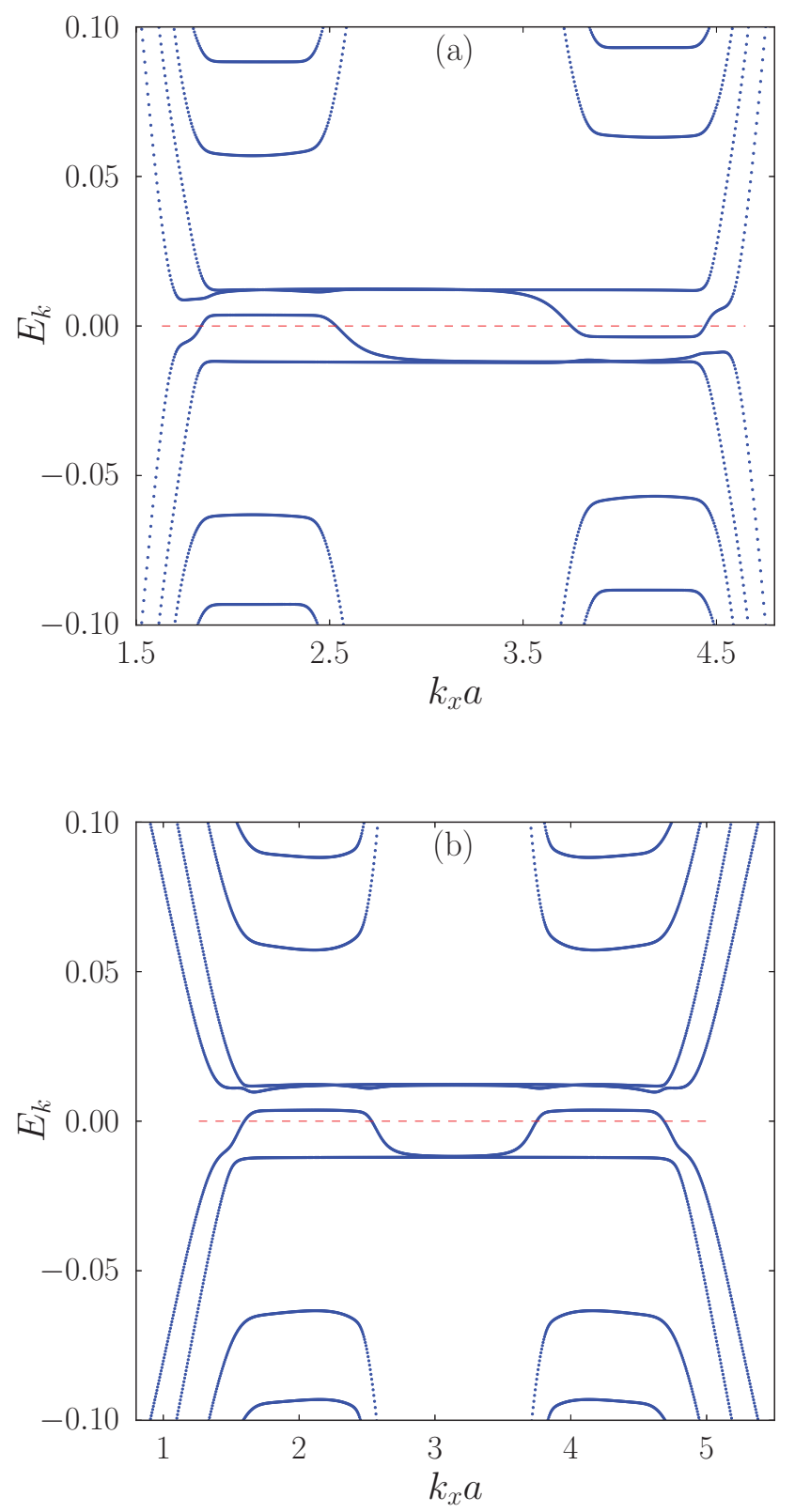

FIG. 5. ( Color online) Landau levels in a biased BLGNR (a) and pseudo Landau levels in an arc-shaped bending of the biased BLGNR (b) for $W=300.5 a_{0}, R=601 a_{0}, l_{B}=23.4 a_{0}$, the bias gate voltage $u=\gamma_{1} / 5$. Dashed red line indicates a constant energy level. Due to the breaking of layer degeneracy by applying bias, the zero Landau level splits into two levels and then higher level shifts in the opposite direction near two valleys when the BLGNR is subjected by the real magnetic field. For the case of pseudo Landau levels there is a mirror symmetry for the energy dispersion of the two valleys and the level shift of pseudo Landau levels is in the same direction in the valleys. 
domagnetic fields. We consider strained BLGNR by deforming it in the arc-shaped bending structure. We calculate the energy spectrum of the system in the presence of a real magnetic field and the electron spectrum is shown in Fig. 4. Decent quantum Hall plateaus are observed mimicking the conventional quantum Hall effect in BLG. The combination of the pseudomagnetic field due to the deformation and a real magnetic field leads to a broadening of all Landau levels except the zero-energy. Moreover, the effective total fields acting on electrons from the valleys $\boldsymbol{K}$ and $\boldsymbol{K}^{\prime}$ are different which results in a valley polarization. As we mentioned before, the pseudomagnetic field picture in BLG is no longer the same as in SLG, because in strained SLG the momentum $\mathbf{p}$ tends to $\mathbf{p}+e \mathbf{A}$, where $\mathbf{A}$ is the fictitious gauge field. In BLG, however, the momentum on interlayer hopping feels like a different pseudo gauge field. Also the energy of PLLs decreases with increasing interlayer vertical hopping consistent with the energy of LLs in BLG which is given by $E_{n}=\frac{2}{\gamma_{1}}\left(\frac{\hbar v_{\mathrm{F}}}{l_{B}}\right)^{2} \sqrt{n(n-1)}$.

It is worthwhile studying the PLLs and LLs in the presence of a perpendicular gate voltage. We calculate the energy dispersions of biased BLGNR in the presence of a real and pseudomagnetic fields and the results are illustrated in Figs. 5a and Fig. 5b, respectively. Due to the breaking of the layer degeneracy by applying the bias gate, the edge state at zero Landau level splits into two levels and then higher level shifts in the opposite direction near to each valley. For the case of PLLs in biased BLGNR, however different features can be occurred which originates from the time reversal symmetry in the mechanically deformed lattice. In this case, there is a mirror symmetry for the energy dispersion of the two valleys and the level shift of PLLs is in the same direction in the valleys. This shift is equal to $\frac{\hbar e u}{2 \gamma_{1}} \xi B_{\xi}$ in which $\xi= \pm$ denotes the valley degree of freedom and $B_{\xi}=\xi\left|B_{p s}\right|$ and thus the PLLs shift equally downward for $u>0$ near to the position of the valleys. The calculated results shown in Fig. 5 are in good agreement with these analysis.

The quantized Hall effect is a generic behavior of 2D electron systems in a strong perpendicular magnetic field. For instance, biased BLGNR in a real magnetic field has $\sigma_{x y}=0$ plateau in Hall measurement which originates from the zero charge transfer ${ }^{47,48}$ across the edge states. We calculate the charge circulation direction for each edge and our numerical results are illustrated in Fig. 6. Charge carriers circulate in opposite directions in two valleys which means that the Hall conductivity of each valley changes in sign and therefore the total Hall conductivity, $\sigma_{x y}$ vanishes in a consistence results with experimental measurements ${ }^{47}$ and theoretical predictions. ${ }^{48}$ However, valley Hall conductivity, which is defined as $\sigma_{x y}^{v}=\sigma_{x y}^{K}-\sigma_{x y}^{K^{y}}$, is finite due to the inversion symmetry breaking. ${ }^{49}$ The non-zero valley Hall conductivity originates from the opposite sign of the Berry curvature in two valleys and it is easy to obtain the Berry curvature as $\Omega_{x y}=-\tau_{z} 2 \Delta a^{4}|q|^{2} /\left(\Delta^{2}+a^{2}|q|^{4}\right)^{3 / 2}$ where $a=\hbar v_{0}^{2} / \gamma_{1}$ where $\Delta=u / 2$. Consequently, in the bi-
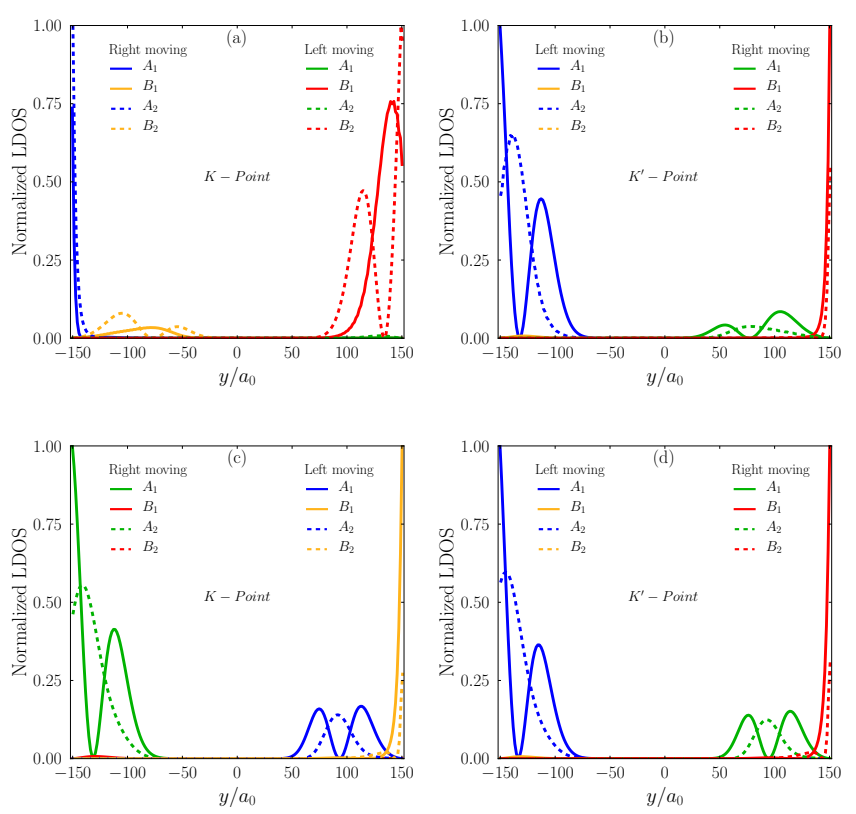

FIG. 6. (Color online) LLs in a biased BLGNR (a, b) and PLLs in the arc-shaped bending of the biased BLG $(c, d)$. The edge states in the gap region are localized on different edges and sublattices for each channel of the edge and electrons in two valleys circulate in opposite directions. Notice that the right moving refers to $\left(A_{i}, B_{1}\right)$ and the left moving denotes to $\left(B_{i}, A_{1}\right)$ sublattices at the Dirac points, $\boldsymbol{K}$ and $\boldsymbol{K}^{\prime}$ respectively, $i=2$ for the real magnetic and $i=1$ for the pseudomagnetic fields. In biased BLGNR subjected by the real magnetic field, a quantum valley Hall effect is expected rather than the quantum Hall effect when the Fermi energy is in the band gap.

ased BLGNR which is subject to the real magnetic field, a quantum valley Hall effect is expected rather than the quantum Hall effect when the Fermi energy is in the band gap. Notice that the state of the system is either in the quantum Hall $(\mathrm{QH})$ phase or in the valley polarized quantum Hall (VPQH) phase for higher Landau levels. The VPQH actually occurs because of the different shifts of LLs around the two valleys. In strained biased BLG, on the other hand, pseudomagnetic field gives different features for LDOS where layer polarization is obtained and the system is in a quantum valley Hall (QVH) phase when the Fermi energy cuts four edge modes as it is shown in Fig. 5. Accordingly, the VPQH phase is absent in the pseudomagnetic field case which preserves the time reversal symmetry. For higher energy, there is a small interval with a trivial insulating gap, without any accusable bulk and edge states, and then system tends to the QVH insulator phase due to different sign of the pseudomagnetic field at two valleys.

In strained graphene the energy band structure is deeply influenced by the form and the value of hopping correction. We show in Fig. 7 that the interlayer spacing has a significant effect on the energy dispersion of 

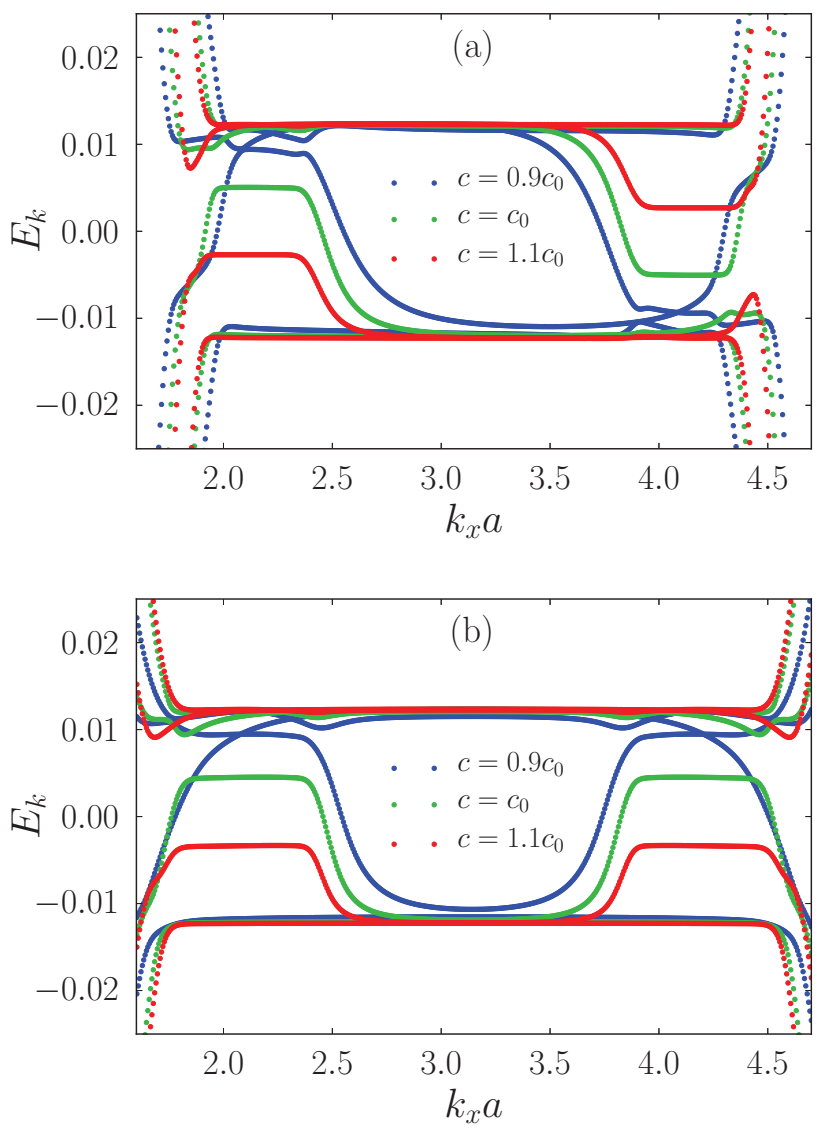

FIG. 7. (Color online) LLs in a biased BLGNR (a) and PLLs in the arc-shaped bending of the biased BLG (b) for different values of the interlayer hoping integral for $W=300.5 a_{0}$, $u=\gamma_{1} / 5, R=781.3 a_{0}$ and $l_{B}=23.2 a_{0}$. The energy dispersion of biased BLGNR profoundly depends on the interlayer spacing which mainly changes the magnitude of $\gamma_{1}$. For a real magnetic field, the large and small values of the interlayer spacing have two different forms of the edge states for zero Landau level. There is a crossed edge states inside the band gap for a larger value of the interlayer, while a crossing disappears and there is a valley polarization for the case that the interlayer hopping is small. There is a topological phase transition from QVH to VPQH and trivial insulator phase by tuning the intralayer spacing at a certain value of Fermi energy. The ratio of $B / \gamma_{1}$ where $B$ is the real magnetic field is the tuning parameter. In pseudomagnetic field, on the other hand, the VPQH phase is absent.

a biased BLGNR and hence we study this effect in the presence of a real and pseudomagnetic fields. The energy dispersion of the biased BLGNR depends strongly on the interlayer spacing which mainly changes the magnitude of $\gamma_{1}$. For a real magnetic field, the large and small values of the interlayer spacing have two different forms of edge states for zero Landau level. There is a crossed edge states inside the band gap ${ }^{47}$ for larger value of the interlayer, while the crossing disappears and there is a valley polarization $(\mathrm{VP})^{32}$ for the case that the interlayer hop-
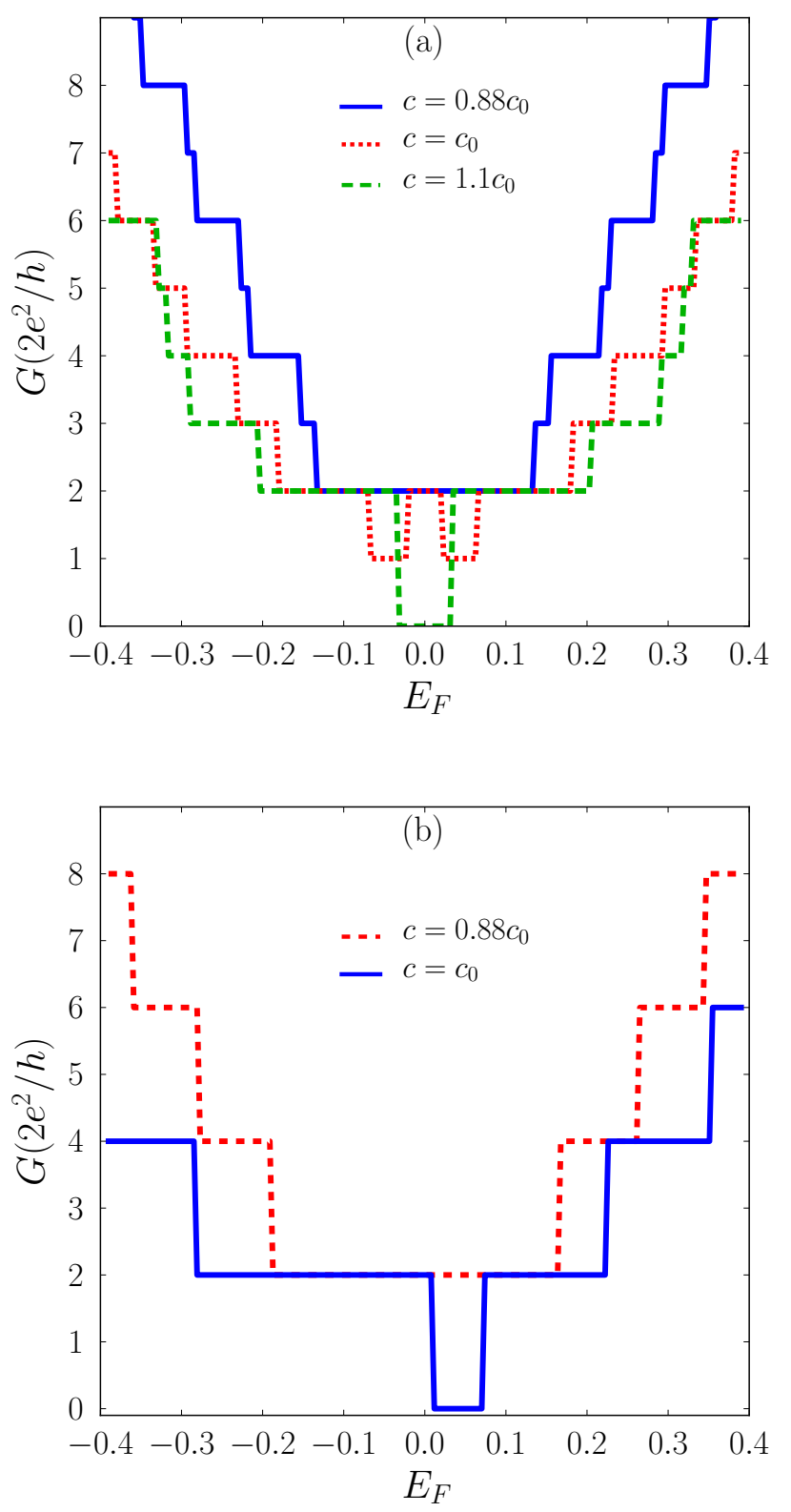

FIG. 8. (Color online) Conductance for different phases in real (a) and pseudomagnetic field (b) for $W=150.5 a_{0}, R=$ $496.6 a_{0}, l_{B}=21.3 a_{0}$ and $u=\gamma_{1} / 2$. The energy is measured in units of $\mathrm{eV}$.

ping is small. Here we demonstrate that there is a topological phase transition from QVH to VPQH and trivial insulator phase by tuning the intralayer spacing at a certain value of the Fermi energy. This feature is shown in Fig. 7a. Note that the ratio of $\alpha=B / \gamma_{1}$ where $B$ is the real magnetic field is the tuning parameter. The reason for that is the fact that the zero Landau level depends on the magnetic field ${ }^{32}, E_{0}(\alpha)$. These results are interesting because the system reveals three different phases inside the band gap for different values of the interlayer spacing. 

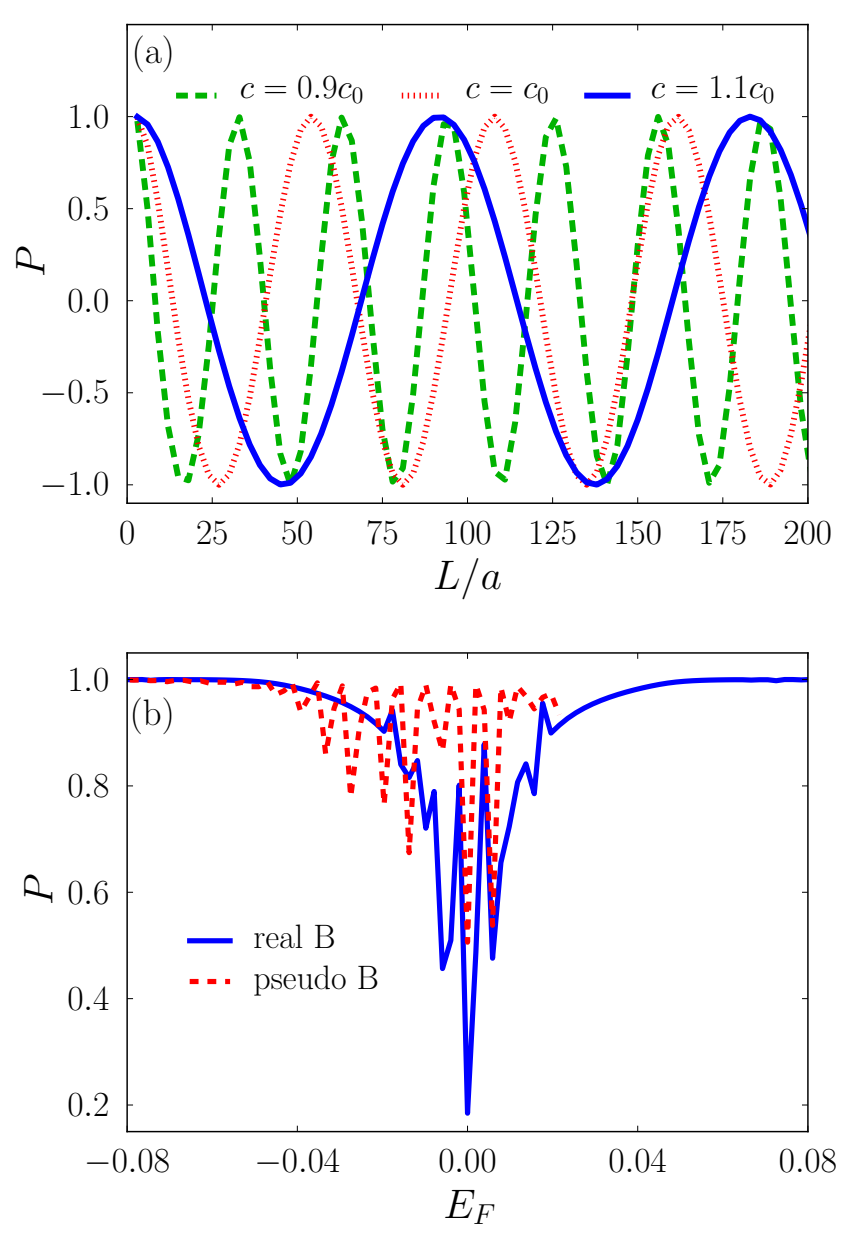

FIG. 9. (Color online) (a) Layer polarization as a function of channel length for different interlayer spacing at real and pseudomagnetic field for $W=75.5 a_{0}, u=0, E=0.01 \mathrm{eV}$ and $a=\sqrt{3} a_{0}$. The layer-resolved transport shows a periodic behavior of the polarization without decaying manner. The period of the oscillation can be tuned by the interlayer spacing and the period increases with increasing $c$ values. (b) Layer polarization as a function of the Fermi energy for biased BLGNR in the presence of a real and pseudomagnetic field for $W=150.5, R=451.5 a_{0}, l_{B}=20.3 a_{0}, L=200 a$, $u=\gamma_{1} / 2$ and $c=c_{0}$. The energy is measured in scale of $\mathrm{eV}$. In the trivial insulator phase there is no transport channel. In the VPQH phase, on the other hand, $P \approx 1$ and it indicates layer-conserved transport. Also the polarization in the QVH phase in the presence of pseudomagnetic field and the vertical bias is higher than the value obtained in the QVH in the real magnetic field.

In pseudomagnetic field, on the other hand, the VPQH phase is absent due to the presence of time reversal symmetry and corresponding numerical results are displayed in Fig. 7b.

We calculate the conductance corresponding to the different phases and our numerical results are shown in Fig. 8. The conductance indicates the number of the transport channel in a two terminal setup. In the pres- ence of the real magnetic field plateaus are even integer values for $\mathrm{QH}$ and $\mathrm{QVH}$ phases however there is an odd integer for VPQH phase due to the breaking of the valley degeneracy. For the pseudomagnetic field case, the valley degeneracy preserves in order that plateaus are even integer. For both the real and pseudomagnetic fields two terminal conductance is zero in the trivial insulating phase due to the absence of any modes to carry any kinds of current.

Moreover, in order to investigate the effect of the interlayer spacing in detail we study the layer-resolved transport properties of BLGNR by calculating $P$ as a quantity which indicates a spectrum from fully layer-conserved $(P=1)$ to fully layer-flip $(P=-1)$ transport. In order to do so, we calculate the polarization as a function of channel length and thus Fig. 9a shows a periodic behavior of the polarization similar to its spin-analogue ${ }^{33}$ without showing a decaying manner. The reason for that is related to the absence of scattering centers in the ballistic transport. It is obvious that the period of the oscillation can be tuned by the interlayer spacing and the period increases with increasing the interlayer spacing values, $c$. Consequently, strain can tune the layer-resolved features in BLGNR. Furthermore, we study the layer-resolved characteristics of BLGNR in the different phases and the numerical results are shown in Fig. 9b. Note that in the trivial insulator phase there is no transport channel so that the polarization does not have any physical meaning. In the $\mathrm{VPQH}$ phase, on the other hand, $P \approx 1$ and it indicates that the layer-conserved transport because, as it is shown in Fig. 6, the right moving transport channel for zero Landau level edge states are mainly localized on different layers for the two valleys. While, in the QVH phase, resulting from the real magnetic field and vertical gate voltage, the polarization reduces although the transport is mainly due to the layer-conserve case. Also the polarization in the QVH phase in the presence of the pseudomagnetic field and the vertical bias is higher than the value in the QVH for the real magnetic field case. This feature can be understood by looking at the LDOS presented in Fig. 6 in which the right moving edge states are mostly localized in the bottom layer for the zero pseudo Landau level. There is the trivial insulator phase inside the gap for $E_{\mathrm{F}}>0.02 \mathrm{eV}$ and the pseudomagnetic case for which transport channels are absented. Indeed, in our special lead structures, the resonating feature of the polarization and conductance comes from the hopping mismatch between the leads and the device region too. This mismatch is a source of a quantum interference effect such as FabryPérot resonances. ${ }^{50}$

\section{CONCLUSION}

We have analyzed in detail the electronic properties of bilayer graphene by putting together the effects of deformations, real magnetic field and perpendicular gate voltage by modifying a lattice model Hamiltonian. We 
have proposed a lattice model Hamiltonian to explore bilayer graphene nanoribbon under the combined effect of deformations, real magnetic fields and gate voltages. The lattice model Hamiltonian, which we have proposed, provides a quite good description of the edge states and we have shown that the pseudo Landau levels in the bulk are no longer dispersive. We have considered a strained bilayer graphene nanoribbon by deforming it in the arcshaped bending structure in the presence of a real magnetic field and found that the zero-energy is chiral. Moreover, the effective total fields acting on electrons from the two-valleys are different which results in a valley polarization.

We have studied the edge states inside the band gap in the presence of a real magnetic field and explored a valley polarized quantum Hall effect, which occurs due to the different shifts of Landau levels around the two valleys, in biased bilayer graphene nanoribbon systems. We have also demonstrated that, in biased bilayer graphene which is subjected to a real magnetic field, the energy dispersion depends on the interlayer spacing and a topological phase transition from quantum valley Hall to valley polarized quantum Hall can occur by tuning the interlayer spacing between two layers. The valley polarized quantum Hall goes toward to a trivial insulator phase with decreasing of the interlayer spacing. In strained biased bilayer graphene, on the other hand, the system is in a quantum valley Hall phase.

The numerical calculations that we have employed to carry out the conductance is the recursive Green's function. Based on that, we have investigated the effect of the interlayer spacing on the layer-resolved transport in bilayer graphene by using layer polarization and its value confirms our obtained phases. We show that the layer polarization in the presence of a pseudomagnetic field is larger than that expected for the value obtained in the presence of the real magnetic filed. We have found that the plateaus of the conductance are even integer values for quantum Hall and quantum valley Hall phases however there is an odd integer for valley polarized quantum Hall phase due to the breaking of the valley degeneracy in the presence of the real magnetic field. For the pseudomagnetic field case, the valley degeneracy preserves in order that plateaus are even integer. It would be interesting to investigate all mentioned phases in the presence of the electron-electron interactions. Valley polarization quantum Hall phase and layer polarization physics, in such way that we proposed, can have very important implications on the electronic properties of bilayer graphene ribbons and other nanostructures.

\section{ACKNOWLEDGMENTS}

R. A. would like to thank the international center for theoretical physics, ICTP, for its hospitality during the period when the last part of this work was carried out. We thank A. Naji for his useful comments.
* asgari@ipm.ir

1 C. Berger, Z. Song, T. Li, X. Li, A. Y. Ogbazghi, R. Feng, Z. Dai, A. N. Marchenkov, E. H. Corad and P. N. First, J. Phys. Chem. B 108, 19912 (2004).

${ }^{2}$ K. S. Novoselov, A. K. Geim, S. V. Morozov, D. Jiang, M. I. Katsnelson, I. V. Grigorieva, S. V. Duboson and A. A. Firsov, Nature (London) 438, 197 (2005).

3 Y. Zhang, Y.-W. Tan, H. L. Stormer and P. Kim, Nature (London) 438, 201 (2005).

4 Taisuke Ohta, Aaron Bostwick, Thomas Seyller, Karsten Horn, Eli Rotenberg, Science 313, 951 (2006); J.B. Oostinga, H.B. Heersche, X. Liu, A.F. Morpurgo, L.M.K. Vandersypen, Nature Mater. 7, 151 (2008); W. Yao, D. Xiao, and Q. Niu, Phys. Rev. B 77, 235406 (2008).

${ }^{5}$ K. S. Novoselov, E. McCann, S. V. Morozov, V. I. Faĺko, M. I. Katsnelson, U. Zeitlet, D. Jiang, F. Schedin and A. K. Geim, Nature physics 2, 177 (2006).

${ }^{6}$ O. Vafek, Phs. Rev. B 82, 205106 (2010); F. Zhang, H. Min, M. Polini and A. H. MacDonald, Phys. Rev. B 81, 041402 (2010).

7 E. McCann and V. I. Faĺko, Phys. Rev. Lett. 96, 086805 (2006); Ya-Fen Hsu, Guang-Yu Guo, Phys. Rev. B. 82, 165404 (2010).

8 Kin Fai Mak, Chun Hung Lui, Jie Shan, Tony F. Heinz, Phys. Rev. Lett.102, 256405(2009).

9 A. B. Kuzmenko, I. Crassee, D. van der Marel, P. Blake and K. S. Novoselov, Phys. Rev. B 80, 165406 (2009).
10 H. Suzuura and T. Ando, Phys. Rev. B 65, 235412 (2002).

11 J. L. Mañes, Phys. Rev. B 76, 045430 (2007).

12 M. A. H. Vozmediano, M. I. Katsnelson, and F. Guinea, Phys. Rep. 496, 109 (2010).

13 F. Guinea, Solid State Commun. 152, 1437 (2012).

14 N. Abedpour, R. Asgari and F. Guinea, Phys. Rev. B 84, 115437 (2011).

15 N. Levy, S. A. Burke, K. L. Meaker, M. Panlasigui, A. Zettl, F. Guinea, A. H. Castro Neto, and M. F. Crommie, Science 329, 554 (2010).

16 H. Rostami and R. Asgari, Phys. Rev. B 86, 155435 (2012).

17 M. M-KruczySnski, I. L. Aleiner and V. I. Faĺko, Phys. Rev. B 84, 041404(R) (2011).

18 D. A. Gradinar, H. Schomerus and V. I. Faĺko, Phys.Rev.B 85, 165429 (2012).

19 Seon-Myeong Choi, Seung-Hoon Jhiand Young-Woo Son, Nano Lett. 10, 3486 (2010).

20 Jen-Hsien Wong, Bi-Ru Wu, Ming-Fa Lin, J. Phys. Chem.C 116, 8271 (2012).

21 Hassan Raza and Edwin C Kan, J. Phys. : Condens. Matter 21, 102202 (2009)

22 B. R. K. Nanda and S. Satpathy, Phys. Rev. B 80, 165430 (2009).

23 E. Mariani, A. J. Pearce and F.v. Oppen Phys. Rev. B 86, 165448 (2012).

${ }^{24}$ Wei Yan, et al., arXiv:1206.5881 (2012).

25 R. Zhao, Y. Zhang, T. Gao, Y. Gao, N. Liu, L. Fu, and Z. 
Liu, Nano Res. 4, 712 (2011); G. Li, A. Luican, J. M. B. Lopes dos Santos, A. H. Castro Neto, A. Reina, J. Kong, and E. Y. Andrei, Nature Phys. 6, 109 (2009); J. Hass, W. A. d. Heer, and E. H. Conrad, J. Phys. Condens. Matter 20, 323202 (2008); D. L. Miller, K. D. Kubista, G. M. Rutter, M. Ruan, W. A. de Heer, P. N. First, and J. A. Stroscio, Phys. Rev. B 81, 125427 (2010); D. L. Miller, K. D. Kubista, G. M. Rutter, M. Ruan, W. A. de Heer, M. Kindermann, P. N. First, and J. A. Stroscio, Nature Phys. 6, 811 (2010).

26 A. H. MacDonald, P. Středa, Phys. Rev. B 29, 1616 (1984).

27 For more details see D. S. L. Abergel, V. Apalkov, J. Berashevich, K. Ziegler and T. Chakraborty, Advances in Physics, 59, 261 (2010).

${ }^{28}$ D. Pesin and A. H. MacDonald, Nature Materials 11,409 (2012).

29 P. San-Jose, E. Prada, E. McCann and H.S chomerus, Phys. Rev. Lett. 102, 247204 (2009).

30 Xiaoguang Li, Zhenyu Zhang, and Di Xiao, Phys. Rev. B 81, 195402 (2010).

31 I. Snyman and C. W. J. Beenakker, Phys. Rev. B 75, 045322 (2007).

32 V. Mazo, E. Shimshoni, H. A. Fertig, Phys. Rev. B 84, 045405 (2011).

33 T. P. Pareek and P. Bruno, Phys. Rev. B 65, 241305(R) (2002).

34 G. Trambly de Laissardiere, D. Mayou and L. Magaud, Nano Lett. 10, 804 (2010).

35 Z. F. Wang, F. Liu and M. Y. Chou, Nano Lett. 12, 3833 (2012).

36 J. C. Slater and G. F. Koster, Phys. Rev. 94, 1498 (1954).
37 P. Moon and M. Koshino, Phys. Rev. B 85, 195458 (2012).

38 A. H. Castro Neto and F. Guinea, Phys. Rev. B 75, 045404 (2007).

39 Vitor M. Pereira and A. H. Castro Neto, and N. M. R. Peres, Phys. Rev. B 80, 045401 (2009).

${ }^{40}$ V. K. Dugaev and M. I. Katsnelson, Phys. Rev. B 86, 115405 (2012).

41 A. Svizhenko, M. P. Anantram, T. R. Govindan, B. Biegel and R. Venugopal, J. Appl. Phys. 91, 2343 (2002).

42 M. P. Lopez Sancho, J. M. Lopez Sancho, and J. Rubio, J. Phys. F: Met. Phys. 14, 1205 (1984); 15, 851 (1985).

43 H. Hatami, N. Abedpour, A. Qaiumzadeh and R. Asgari, Phys. Rev. B 83, 125433 (2011).

44 T. Low and F. Guinea, Nano Lett. 10, 3551 (2010).

45 B. Roy, Z-X. Hu, and K. Yang, Phys. Rev. B 87, 121408(R) (2013).

46 V. Lukose, R. Shankar, and G. Baskaran, Phys. Rev. Lett. 98, 116802 (2007).

47 Eduardo V. Castro, K. S. Novoselov, S. V. Morozov, N. M. R. Peres, J. M. B. Lopes dos Santos, Johan Nilsson, F. Guinea, A. K. Geim, and A. H. Castro Neto, Phys. Rev. Lett. 99, 216802 (2007).

${ }^{48}$ R. Ma, L. J. Zhu, L. Sheng, M. Liu and D. N. Sheng, Eur. Phys. Lett. 87, 17009 (2009).

49 D. Xiao, W. Yao, and Q. Niu, Phys. Rev. Lett. 99, 236809 (2007).

50 Y. V. Nazarov and Y. M. Blanter Quantum Transport:Introduction to Nanoscience, Cambridge University Press (2009). 